\title{
Digital Platform for the Optimization of Occupational Health and Safety Systems Specific to the Industrial Area - Part I
}

\author{
Petru Iulian MUREȘAN \\ Transilvania University of Brasov, Romania, petru.muresan@unitbv.ro \\ Ioan MILOSAN \\ Transilvania University of Brasov, Romania, milosan@unitbv.ro \\ Adina CHIRIL $\breve{A}$ \\ Transilvania University of Brasov, Romania, adina.chirila@unitbv.ro
}

\begin{abstract}
In Romania there was a long period of time in which the activity of the occupational health and safety system (OHS) was regulated. Over the time, it became mandatory to fill in the instruction material exclusively by hand. As a result, it proves to be absolutely necessary to optimize the activity of the process of management of the documents corresponding to occupational health and safety systems. This was done by means of our online application SSM.RO. The purpose of the study is to evaluate the efficiency and the accessibility level of this application. The main method was a questionnaire that was applied to 246 respondents. The results of this questionnaire were analyzed by means of Fisher's exact test. The most statistically significant association was observed between the highest successfully completed level of education (which can be university or high school) and the level of accessibility of the application SSM.RO. We also observed a relationship between the highest successfully completed level of education of the employees and the way in which the employees acquired knowledge for the use of the Internet. It is very important that more than $91 \%$ of the respondents expressed their willingness to have the future trainings in an online environment since the application SSM.RO was considered intuitive and easy to use.
\end{abstract}

Keywords

digital platform, optimization, occupational health and safety

\section{Introduction}

Recently, we observed that many researchers published papers on the importance of digital platforms [1-4], their benefits for entrepreneurial activities [5-6], their implications for digital business strategies [7]. One of the most prominent directions of research is the use of digital platforms for industrial processes [8-9].

In Romania there was a long period of time in which the activity of the occupational health and safety system (OHS) was regulated. Over the time, it became mandatory to fill in the instruction material exclusively by hand. As a result, it proves to be absolutely necessary to optimize the activity of the process of management of the documents corresponding to occupational health and safety systems.

The optimization of this process should have a large applicability range. Moreover, it should address all the categories of participants who are implied in the work process, namely the employers, the employees, the workers and the public authorities with attributions in the area of the occupational health and safety system, the prevention and fire-fighting staff and the work relationships.

The optimization of the activity of the process of management and administration of the documents corresponding to the occupational health and safety system can be done in an efficient way by the electronic administration of documents (digitizing its services).

\section{Materials and Methods}

\subsection{The website https://www.ssm.ro}

The digital platform SSM.RO (Fig. 1) offers the companies - in particular in the remote process of instruction of the workers - the possibility to electronically sign the documents that are issued and sent to their workers, in an electronic format. In this way, any issued document, which is sent and signed in 
electronic format complies with the requirements required by the legislation in force so that they produce the juridical effects imposed by the Law 319/ 2006, G.D 1425/ 2006, Law 208/ 2021 and the other normative acts in force, which establish norms regarding the occupational health and safety systems. Note that the certificates for remote electronic signature that are being used by the application are issued by a Romanian provider who is qualified for remote electronic signature.

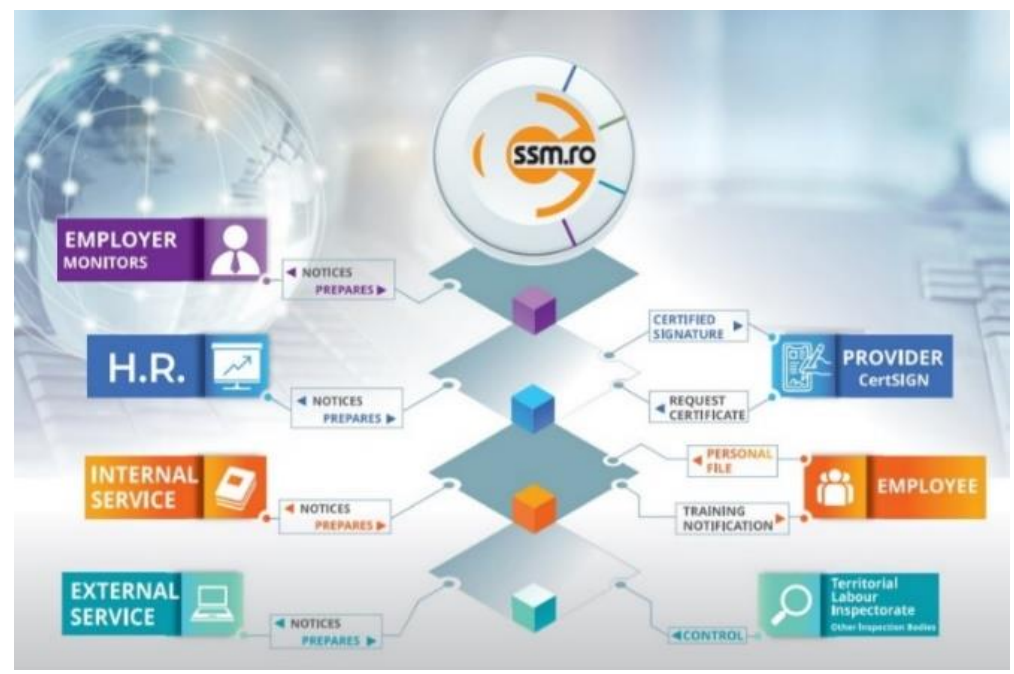

Fig. 1. The structure of the application SSM.RO

The remote learning assumes that the user follows a few steps.

1. Sign in. The user receives an e-mail with the invitation to connect to the application. The user creates an account and has access to the application from anywhere.

2. The user fills in his/her profile with personal data in the account which the company has in the application. The user can be an employee, an OHS inspector or the administrator of a trading company.

3. The upload/ the generation of documents directly in the application.

4. The electronic signature; The documents are validated directly in the application by means of the qualified/ advanced electronic signature together with the creation data and the qualified electronic certificate of the employer.

At the first authentication, once the administrator or the OHS inspector from the company has confirmed his/her account, the following files have to be introduced in the profile of the company:

- OHS/ FSS documents that are specific to the activity of the company, decisions, instructions and evaluations for the workers, which have to be uploaded to the platform in Word of PDF format;

- the Revisal file, which is automatically uploaded with the data of the employees in order to fill in easily their profiles. Before uploading the Revisal file, the person who is responsible with the occupational health and safety systems can edit the fields from the profile of the employees so that their data are as complete as possible. For example, they can add columns such as e-mail address, telephone number, department, function;

- personal questionnaires for instructions in Excel format;

- the organization chart; in order to facilitate learning, we can create from the application the structure of the organization of the company, based on departments.

Once the documents are uploaded, the application can automatically generate the topics, the trainings and the testing questionnaires for the employees. Every employee will receive an e-mail with an invitation to create an account, which has to be confirmed by means of the telephone number. Then, after registration in the account, the employees can go through the training files, can solve the evaluation questionnaires, can sign them electronically at the end of the regular training session. The person who is responsible with the occupational health and safety systems can view the reports with the employees who finished the training. This person and the administrator of the company are notified by the application in order to sign the files of the employees. 
Moreover, the application automatically notifies the user, by e-mail, with regards to the due date for the electronic signature of the documents, the date for the evaluation of the employees or the date for the regular medical examinations.

The application automatically generates reports with regards to: documents that have to be issued for every employee, deadlines for approval and submission, expiry dates for the trainings or any other dates regarding obligations imposed by the law in force.

When we use the application there is no numerical limit for the employees that can be handled by the platform. Whether the application will manage 1 or 10,000 employees, the company that uses the application will benefit to the same extent from all the functionalities of the platform.

\subsection{Questionnaire - practical validation regarding the implementation of the online SSM training of the employees}

The practical validation regarding the implementation of the online training of the employees that refers to the occupational health and security systems has been done by using a case study. More precisely, we conducted experimental research regarding the implementation of the online training for the occupational health and security systems with an approximatively number of 800 employees of some companies that were randomly chosen.

After the effective training that was organized both classical (in the training room) and online, the employees were asked to submit a questionnaire reply, which contains 15 questions that are relevant for the proposed goal (remark: seven of the questions will be presented and analysed in this paper, and the remaining eight questions will be presented and analysed in a future paper).

The questions of the questionnaire are presented below.

1. How often do you use the Internet?

- on a daily basis

- very rarely

- at least once every month

2. Which is your age category?

- 18 - 24 years old

- 25 - 34 years old

- 35 - 44 years old

- 45 - 54 years old

- over 55 years old

3. Which is the highest successfully completed level of education?

- university

- high school

4. The place where you carry out your work is

- in the urban area

- in the rural area

5. The knowledge regarding the use of the Internet were acquired

- by being enrolled in some form of education or in introductory courses regarding the use of the computer

- by individual study

6. On a scale from 1 to 5 , how easy do you think that the access to the online training is? (1 - very hard to access up to 5 - very easy to access)

- 1 - very hard

- 2 - hard

- 3 - medium access

- 4 - easy

- 5 - very easy

7. On a scale from 1 to 5 , how efficient do you think that the online training is? ( 1 - not efficient, up to 5 - very efficient)

- 1 - not efficient

- 2 - little efficient 
- 3 - medium efficiency

- 4 - efficient

- 5 - very efficient

\subsection{Fisher's exact test}

We employ independence tests in order to establish if we have a significant relationship between two categorical variables. We recall two types of independence tests: the Chi-square test and the Fisher's exact test [10-12].

We cannot employ the Chi-square test if in the contingency table we have cells with expected values that are less than 5. However, we can use Fisher's exact test in this case.

Both Fisher's exact test and the Chi-square test have the same hypotheses. Namely, HO tells us that the variables are independent and we have no relationship between them. Moreover, if we have the value of one variable, then we cannot predict the value of the other variable. On the other hand, H1 tells us that the variables are dependent and we have a relationship between them. Furthermore, if we have the value of one variable, then we can predict the value of the other variable.

We are interested in computing the p-value, which is the probability of having observations at least as extreme as the one we measured (via the samples) if the null hypothesis were true. It indicates how likely the null hypothesis is. We can also consider that it is the smallest level of significance for which the data indicate rejection of the null hypothesis. If the p-value is under the threshold of $5 \%$, then we deduce that the observations (and thus the test statistic) are too unlikely to happen just by chance if the null hypothesis were true. Hence, we reject the null hypothesis. On the other hand, if the p-value is above the threshold of $5 \%$, then the null hypothesis is not rejected.

\section{Results}

\subsection{The answers to the questionnaire}

The first question: the frequency in the use of the online environment

The question has the goal to obtain an overall picture regarding how often the employees use the online environment (Fig. 2).

From the summary of the responses, it follows that $97.56 \%$ of the respondents use the online environment on a daily basis and only $2.03 \%$ use the online environment very rarely.

Conclusion: we draw the conclusion that the implementation of a software for the online training of the employees would have a high level of addressability.

The second question: the acquisition of knowledge for the use of the Internet. The goal of the question was to obtain an overall picture regarding the acquisition of knowledge for the use of the Internet by the respondents to the questionnaire. In Figure 3 we present the answers regarding the second question.

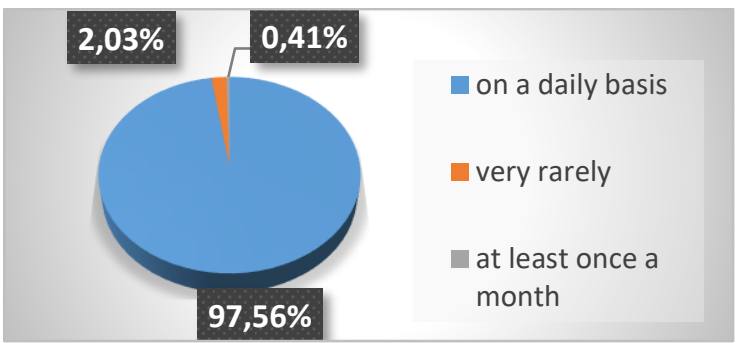

Fig. 2. The criterion of frequency in the use of the online environment

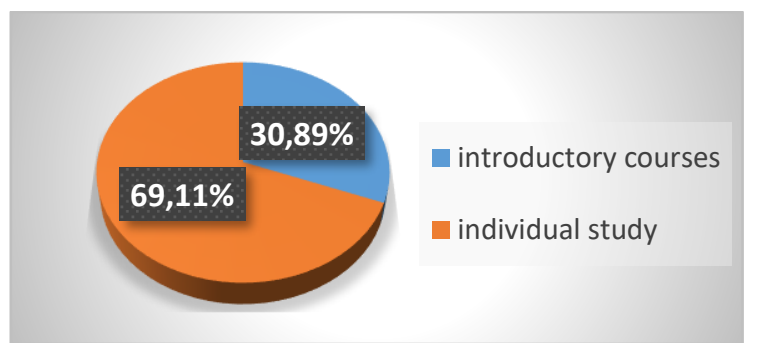

Fig. 3. The criterion regarding the acquisition of knowledge for the use of the Internet

Conclusion: from the summary of the answers, it follows that $69.11 \%$ of the respondents have acquired knowledge for the use of the Internet by individual study and $30.89 \%$ of them were enrolled in some form of education or in introductory courses regarding the use of the computer.

The third question: the level of accessibility to online training

This question has the goal to obtain an overall picture regarding the level of accessibility to online training of the employees. In Figure 4 we present the answers to the third question. 
From the summary of the answers, it follows that for $78.86 \%$ of the respondents the level of accessibility to online training is perceived as very easy and only for $0.41 \%$ of them it is very hard, while for $0.80 \%$ of them it is hard. Conclusion: the online training of the employees implies a high level of accessibility for the majority of the respondents.

The fourth question: the level of efficiency of online training

The question has the goal to obtain an overall picture regarding the level of efficiency of online training for the employees. In Figure 5 we present the answers to the fourth question.

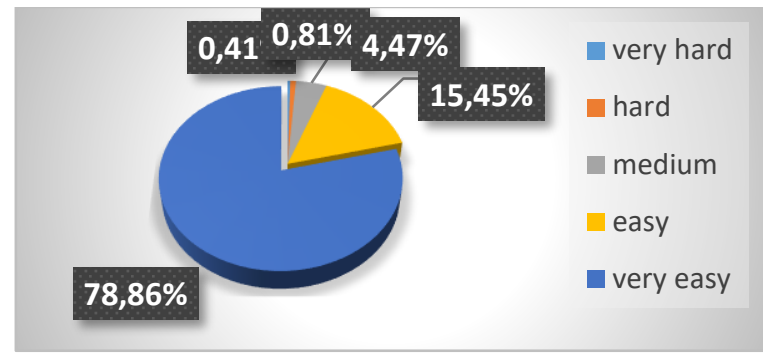

Fig. 4. The criterion regarding the level of accessibility to online training

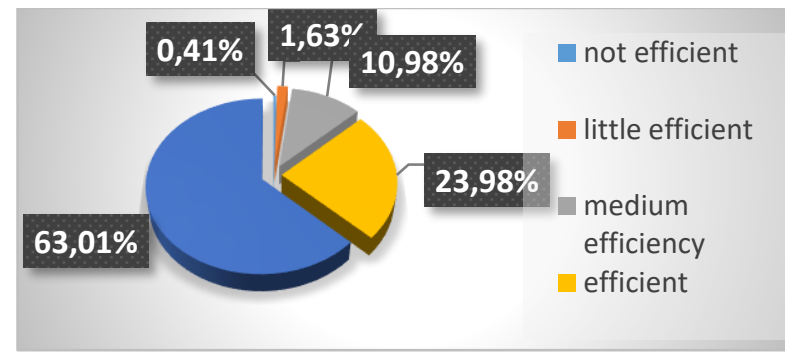

Fig. 5. The criterion regarding the level of efficiency of the online training

From the summary of the answers, it follows that for $63.01 \%$ of the respondents the level of efficiency of the online training is perceived as very efficient and only for $0.41 \%$ of them it is not efficient, while for $1.63 \%$ of them it is little efficient. Conclusion: the online training of the employees implies a high level of efficiency for the majority of the respondents.

\subsection{Fisher's exact test}

In this section we would like to find out if there is a statistically significant association between.

- The age of the employees and the accessibility level to online instruction by means of the application SSM.RO;

- The age of the employees and the level of difficulty in employing the application SSM.RO;

- The area of the employees (urban/rural) and the fact that the online instruction by means of the application SSM.RO is more efficient than other instruction forms.

The data was collected from 246 individuals.

\subsubsection{The age of the employees and the accessibility level to online instruction by means of the application SSM.RO}

First of all, we study the relationship between the age of the employees and the accessibility level to online instruction. The age of the employees is grouped in five categories, namely 18-24, 25-34, 35-44, $45-54,>55$. The accessibility level to online instruction is grouped into five categories, namely 1-very hard, 2-hard, 3-medium, 4-easy, 5-very easy. The relevant code is described in Figure 6.

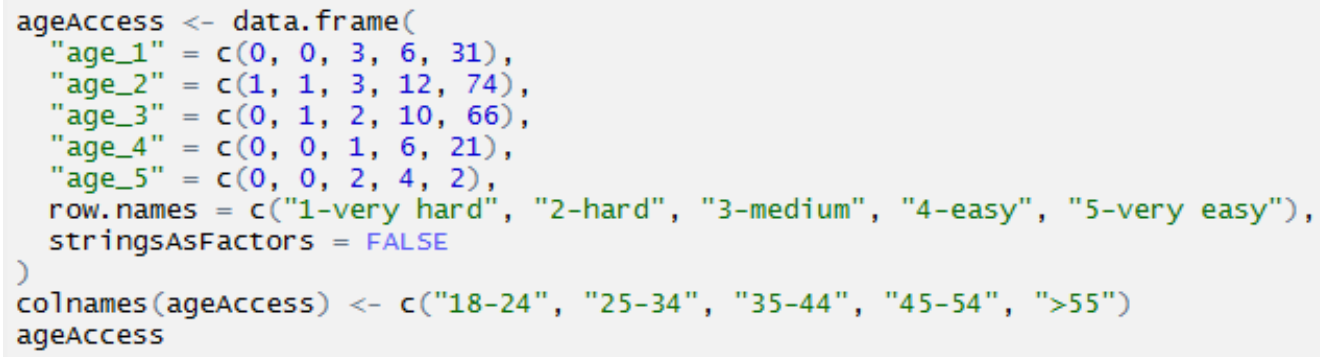

Fig. 6. The code written in R in order to represent the contingency table of the age groups and the level of accessibility to online instruction

We describe the data in a contingency table, as can be seen in Figure 7. 
RECENT, Vol. 22, no. 3(65), 2021

\begin{tabular}{lrrrrr} 
& $\begin{array}{r}18-24 \\
<\mathrm{db} \mid>\end{array}$ & $\begin{array}{r}25-34 \\
\langle\mathrm{db}|>\end{array}$ & $\begin{array}{r}35-44 \\
\langle\mathrm{db}|>\end{array}$ & $\begin{array}{r}45-54 \\
\langle\mathrm{db}|>\end{array}$ & $\begin{array}{r}>55 \\
\langle\mathrm{~d} b|>\end{array}$ \\
\hline 1-very hard & 0 & 1 & 0 & 0 & 0 \\
2-hard & 0 & 1 & 1 & 0 & 0 \\
3-medium & 3 & 3 & 2 & 1 & 2 \\
4-easy & 6 & 12 & 10 & 6 & 4 \\
5-very easy & 31 & 74 & 66 & 21 & 2 \\
5 rows & & & & &
\end{tabular}

Fig. 7. The figure represents the contingency table of the age groups and the level of accessibility to online instruction.

In the following, we compute the expected frequencies, by the following code in R: chisq.test(ageAccess)\$expected. The result is represented in Figure 8.

$\begin{array}{lrrrrr}\text { 1-very hard } & 0.1626016 & 0.3699187 & 0.3211382 & 0.1138211 & 0.03252033 \\ \text { 2-hard } & 0.3252033 & 0.7398374 & 0.6422764 & 0.2276423 & 0.06504065 \\ \text { 3-medium } & 1.7886179 & 4.0691057 & 3.5325203 & 1.2520325 & 0.35772358 \\ \text { 4-easy } & 6.1788618 & 14.0569106 & 12.2032520 & 4.3252033 & 1.23577236 \\ \text { 5-very easy } & 31.5447154 & 71.7642276 & 62.3008130 & 22.0813008 & 6.30894309\end{array}$

Fig. 8. The expected frequencies for the table representing the age groups and the level of accessibility to online instruction.

Note that the contingency table in Figure 8 contains many cells with values below five. This shows us that the Chi-square test may be incorrect, so we should only study the Fisher's exact test. The corresponding code in $\mathrm{R}$ is fisher.test(ageAccess, workspace $=2 \mathrm{e} 7$ ).

In $\mathrm{R}$, this test gives us a p-value of 0.05928 . The $\mathrm{p}$-value is above the threshold of $5 \%$, which means that the null hypothesis is not rejected. This implies that we do not reject that fact that the variables are independent. However, we do not accept the null hypothesis (the fact that there is no relationship between the age of the employees and the level of accessibility to online instruction) since the p-value is very close to the threshold.

3.2.3. The area of the employees (urban/rural) and the fact that the online instruction by means of the application SSM.RO is more efficient than other instruction forms

In this section we study if there is a statistically significant association between the area in which the employees operate (which can be an urban or a rural environment) and the yes/no answers of the employees to the question: is the online instruction by means of the application SSM.RO more efficient than other instruction forms?

First of all, we represent the relevant results of the online questionnaire in a $2 X 2$ contingency table, as can be seen in Figure 9.

\begin{tabular}{lrr} 
& $\begin{array}{r}\text { urban } \\
<\mathrm{db} \mid>\end{array}$ & $\begin{array}{r}\text { rural } \\
<\mathrm{dbl}>\end{array}$ \\
\hline yes & 167 & 26 \\
no & 52 & 1 \\
\hline rows & & \\
\hline
\end{tabular}

Fig. 9. The figure represents the contingency table of the area of the employees and the answer (yes/no) to the question of online instruction being more efficient than other instruction forms

Then we compute the corresponding expected frequencies. Note that all these values are above five (Fig. 10).

$$
\begin{array}{lrr} 
& \text { urban } & \text { rura1 } \\
\text { yes } & 171.81707 & 21.182927 \\
\text { no } & 47.18293 & 5.817073
\end{array}
$$

Fig. 10. The expected frequencies for the table representing the area of the employees and the answer

(yes/no) to the question of online instruction being more efficient than other instruction forms 
Then we perform the Fisher's exact test in R, which gives us the result from Figure 11.

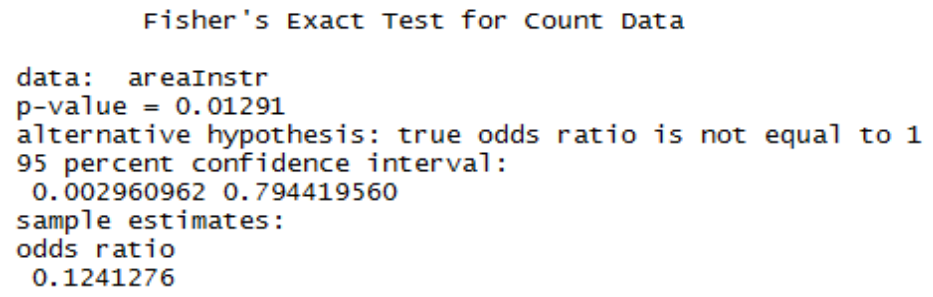

Fig. 11. Fisher's exact test for the table representing the area of the employees and the answer (yes/no) to the question of online instruction being more efficient than other instruction forms

Note that in $\mathrm{R}$ we can find out the confidence interval of the odds ratio. If the confidence interval of the odds ratio includes unity, then we cannot draw the conclusion that there is a difference between the two groups.

An odds ratio of 1 would mean that there is no association between the area of the employees and the opinion that online instruction is more efficient than other instruction forms. If the $95 \%$ confidence interval of the odds ratio does not include 1 , then we can draw the conclusion that the association is significant. This can be seen in Figure 11.

The P-value of 0.01291 shows that we have a $1.291 \%$ risk of falsely concluding a relationship between the data. Note that this value is less than $5 \%$, which is acceptable by convention.

\subsubsection{The highest successfully completed level of education and the acquisition of knowledge for the use of}

the Internet

In this section, we study if there is a relationship between the highest successfully completed level of education of the employees and the way in which the employees acquired knowledge for the use of the Internet (by enrolling in a course or by individual study). The corresponding contingency table appears in Figure 12.

\begin{tabular}{|c|c|c|}
\hline & university & highschool \\
\hline course & 71 & 5 \\
\hline individual study & 135 & 35 \\
\hline
\end{tabular}

Fig. 12. The figure represents the contingency table of the highest successfully completed level of education and the acquisition of knowledge for the use of the Internet

We compute the expected frequencies in the table from Figure 13. Note that all the entries of the table have values above 5 .

$$
\begin{array}{lrr} 
& \text { university } & \text { highschool } \\
\text { course } & 63.64228 & 12.35772 \\
\text { individual study } & 142.35772 & 27.64228
\end{array}
$$

Fig. 13. The expected frequencies for the table representing the highest successfully completed level of education and the acquisition of knowledge for the use of the Internet

Then we perform the Fisher's exact test in R. This can be seen in Figure 14.

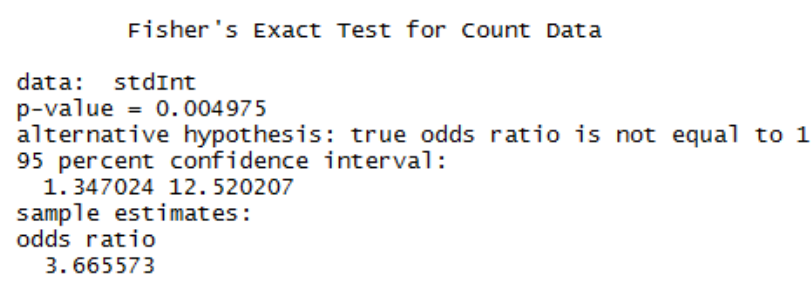

Fig. 14. Fisher's exact test for the table representing the highest successfully completed level of education and the acquisition of knowledge for the use of the Internet 
The p-values is small and below $5 \%$, namely 0.004975 . This shows a significant association between the data. Moreover, the odds ratio is different from 1 and the $95 \%$ confidence interval of the odds ratio does not include unity. This also proves that the association is significant.

\section{Conclusions}

As a consequence of the results of the experimental research regarding the implementation of the online training for the occupational health and security systems, we conclude that the implementation of a software for the online training of the employees has a high level of utility and addressability from the point of view of all the subjective criteria that were analysed for the employees. Moreover, the online training of the employees implies a low level of difficulty for the majority of the respondents.

The Fisher's exact test led us to the conclusion that there is in fact a relationship between the age of the employees and the level of difficulty in employing the application SSM.RO. However, we deduced that there is insufficient evidence to a difference between the age of the employees and the level of accessibility to online instruction. Note that Fisher's exact test is a hypothesis test. It shows whether a difference is likely under the null hypothesis of independence. However, it provides no information about the possible size of the difference.

Taking into consideration the security measures that are imposed at the national level in this time span, the application can offer the possibility for the work inspectors to use the online environment on legal grounds. Therefore, there is no necessity to physically interact with the employer in order to make available the documents that are necessary for performing the control. Moreover, there is no possibility that the COVID-19 virus can be transmitted by means of these documents.

\section{References}

1. Bonina C., Koskinen K., Eaton B., Gawer A. (2021): Digital platforms for development: Foundations and research agenda. Information Systems Journal, ISSN 1365-2575, Vol. 31, special issue paper, pp. 869-902, https://doi.org/10.1111/isj.12326

2. Alaimo C., Kallinikos J., Valderrama E. (2020): Platforms as service ecosystems: Lessons from social media. Journal of Information Technology, ISSN 1466-4437, Vol. 35, No. 1, pp. 25-48, https://doi.org/10.1177/ $\underline{0268396219881462}$

3. de Reuver M., Sørensen C., Basole R.C. (2018): The digital platform: A research agenda. Journal of Information Technology, ISSN 1466-4437, Vol. 33, No. 2, p. 124-135, https://doi.org/10.1057/s41265-016-0033-3

4. Constantinides P., Henfridsson O., Parker G.G. (2018). Introduction - Platforms and infrastructures in the digital age. Information Systems Research, ISSN 1526-5536, Vol. 29, No. 2, pp. 381-400, https://doi.org/10.1287/ isre.2018.0794

5. Nambisan S. (2017): Digital Entrepreneurship: Toward a Digital Technology Perspective of Entrepreneurship. Entrepreneurship Theory \& Practice, ISSN 1042-2587, Vol. 41, is. 6, pp 1029-1055, https://doi.org/10.1111/ etap.12254

6. Srinivasan A., Venkatraman N. (2018): Entrepreneurship in Digital Platforms: A Network-Centric View. Strategic Entrepreneurship Journal, ISSN 1932-443X, Vol. 12, No. 1, pp. 54-71, https://doi.org/10.1002/sej.1272

7. Markus L.M., Loebbecke C. (2013): Commoditized Digital Processes and Business Community Platforms: New Opportunities \& Challenges for Digital Business Strategies. MIS Quarterly, ISSN 2162-9730, Vol. 37, No. 2, p. 649-653, https://www.jstor.org/stable/43825930

8. Savastano M., Amendola C., Bellini F., D’Ascenzo F. (2019): Contextual Impacts on Industrial Processes Brought by the Digital Transformation of Manufacturing: A Systematic Review. Sustainability, ISSN 2071-1050, Vol. 11(3), article 891, https://doi.org/10.3390/su11030891

9. Johansson N., Roth E., Reim W. (2019): Smart and Sustainable eMaintenance: Capabilities for Digitalization of Maintenance. Sustainability, ISSN 2071-1050, Vol. 11(13), article 3553, https://doi.org/10.3390/su11133553

10. Agresti A. (2012): Categorical Data Analysis. 3rd ed., Wiley, ISBN 978-0-470-46363-5, pp. 90-103

11. Dahiru T. (2008): P-value, a true test of statistical significance? A cautionary note. Annals of Ibadan Postgraduate Medicine, ISSN 1597-1627, Vol. 6, No. 1, pp. 21-26, DOI: 10.4314/aipm.v6i1.64038

12. McCrum-Gardner E. (2008): Which is the correct statistical test to use? BRITISH Journal of Oral and Maxillofiacial Surgery, ISSN 0266-4356, Vol. 46(1), pp. 38-41, https://doi.org/10.1016/j.bjoms.2007.09.002 